\title{
Immunohistochemical Investigations of Parvalbumin Localization in the Skeletal Muscle Fibers of Rats
}

\author{
By
Hiromichi ANNOH, Tetsuo INOKUCHI, Keiske OHTA, Mitsugu WAKIMOTO and
Tamako UEDA

Department of Anatomy, Kurume University School of Medicine, Asahi-machi 67, Kurume-shi, Fukuoka 830 Japan

- Received for Publication, September 4, $1995-$

Key Words: Parvalbumin, Skeletal muscle, Rat, Immunohistochemistry, Troponin

\begin{abstract}
Summary: Parvalbumin (PA) is a water soluble, low-molecular weight, calcium-binding protein which has been thought to be involved in the relaxation of skeletal muscle fibers. Although it is well known that PA concentrations are higher in fast twitch fiber than slow twitch fiber, the localization of PA within the cytoplasm of single muscle fibers is still unknown. The present study, therefore, was undertaken to clarify the PA localization by immunohistochemical methods using the confocal laser scanning microscope (CLSM) and transmission electron microscope (TEM).

Wistar strain male rats were fixed by vascular perfusion with $4 \%$ paraformaldehyde solution, and the extensor digitorm longus muscle was dissected out. For fluorescent antibody examination, these specimens were quickly frozen in melting isopentan and sections were cut using a cryostat at $-25^{\circ} \mathrm{C}$. These sections were incubated in anti-PA and antiTroponin (TR) respectively, and then exposed to Texas-Red- and FITC-labelled secondary antibodies. For TEM study, the pre-embedding method was used. Fluorescent immunohistochemical study has clearly shown that both PA and TR are located intimately in the I-band of the skeletal muscle fibers. The finding by the immunofluorescent study correlated well with those which have been seen at the ultrastructural level. The fact that PA is located in close proximity to TR is considered to be very reasonable when we consider it in terms of the muscular contraction-relaxation cycle.
\end{abstract}

Parvalbumin (PA), a highly water soluble, lowmolecular weight, cytosolic $\mathrm{Ca}^{2+}$ binding protein, has been detected in skeletal muscle fibers of not only lower vertebrates, but animals, and has been thought to be involved in the muscular relaxation process ${ }^{1 \sim 3,21}$. It has been thought that the contraction-relaxation cycle of the skeletal muscle is performed as follows; in the relaxed state, $\mathrm{Ca}^{2+}$ within the cytoplasm of a single muscle fiber locates preferentially in the sarcoplasmic reticulum (SR) and the PA is saturated with $\mathrm{Mg}^{2+}$. When the skeletal muscle fibers are about to contract, $\mathrm{Ca}^{2+}$ are released from the SR to the intermyofibrillar space and then $\mathrm{Ca}^{2+}$ binds to troponin (TR)-C which is located in close association with actin filaments, leading to the myofibrillar contraction. Following the dissociation of $\mathrm{Mg}^{2+}$ from $\mathrm{PA}, \mathrm{Ca}^{2+}$ is transferred from TR to PA causing the relaxation. $\mathrm{Ca}^{2+}$ is, thereafter, taken into the SR and PA is, on the other hand, rebound with $\mathrm{Mg}^{2+1-3)}$. It is suggested that the muscular relaxation speed will be influenced by the PA concentration within the cytoplasm of the muscle fiber ${ }^{3)}$. In fact, the concentration of this protein is higher in fast-twitch muscle than in slow-twitch muscle ${ }^{4)}$. Celio and Heizmann ${ }^{14)}$ immunohistochemically investigated the skeletal muscle fiber of the rat and reported that type IIB muscle fiber reacted very strongly with anti-PA antibody, moderately or weakly with type IIA fibers, and scarcely with type I fibers in a cross section of rat skeletal muscle fibers. Schmitt and Pette ${ }^{17)}$ have quantitatively measured the amount of PA in single fibers of rabbit skeletal muscle and defined their respective histochemical myofibrillar ATPase activities, and they have reported the presence of a higher concentration of PA in type IIB fibers than in the other types of fibers. Thus, the distribution of PA in different muscular fiber types is relatively well elucidated. However, the more precise localization of PA within the cytoplasm of the individual muscle fiber is still unknown. The present study has been undertaken, therefore, in order to clarify the PA localization in the longitudinal and cross section of skeletal muscle fibers of rats by means of immunohistochemical methods using confocal laser scanning microscope (CLSM) and also transmission electron microscope (TEM). 


\section{Materials and methods}

Adult male Wistar strain rats $(200 \sim 250 \mathrm{~g})$ were used in this study. After the animals were anesthetized with ketamine hydrochloride and were vascular-perfused with $4 \%$ paraformaldehyde solution (phosphate buffer, $\mathrm{pH} 7.2$, room temperature) through the canulated aorta, the extensor digitorm longus muscles were quickly dissected out. These muscles were immersed in $4 \%$ paraformaldehyde solution, as above, at $4^{\circ} \mathrm{C}$ overnight. The specimens were then washed in phosphate buffered saline (PBS) at $4^{\circ} \mathrm{C}$, and later transferred into PBS containing $7.5 \%, 10 \%$ and $15 \%$ saccharose in turn. The specimens were quickly frozen in melting isopentan and stored at $-85^{\circ} \mathrm{C} .10 \mu \mathrm{m}$ thick longitudinal and cross sections of muscles were cut using a cryostat at $-25^{\circ} \mathrm{C}$, attached to poly-L-lysin-coated slide-glasses and air-dried for $30 \mathrm{~min}$. Sections were incubated for $60 \mathrm{~min}$ in $10 \%$ normal goat serum in order to block nonspecific antibody binding sites. After washing five times ( $3 \mathrm{~min}$ each) in PBS containing $0.03 \%$ Triton-X-100 (PBS-T), the sections were incubated with primary antibody (monoclonal, anti-PA IgG $_{1}$, host; mouse, Sigma, USA) (1:2000, diluted in PBS) at $4^{\circ} \mathrm{C}$ for two days. After washing in PBS-T, sections were incubated with secondary antibody (anti-mouse IgG $_{1}$ biotinilated whole antibody, Amersham, Great Britain) (1:200, diluted in PBS) overnight. Thereafter, sections were washed in PBS-T five time for $5 \mathrm{~min}$ each time, reacted to Texas-Red-labelled streptavidin (Life Technologies Inc., USA) (1:200, diluted in PBS), and were washed in PBS. Several sections were further incubated in another antibody (Polyclonal, anti-TR-T $\operatorname{IgG}_{1}$, host; rabbit, Oncogen Sci., USA) (1:1000, diluted in PBS), following the reaction with FITC-labelled secondary antibody (anti-rabbit $\mathrm{IgG}_{1}$ ). Sections were mounted with glycerin containing $1 \% \mathrm{DABCO}$ and were examined by CLSM (Carl Zeiss, 310UV).

Transmission electron microscopic (TEM) study was also performed. The pre-embedding method was used for the TEM study. Thin, small fragments of muscle fibers were reacted with the same primary antibody as described above, at $4^{\circ} \mathrm{C}$ for two days. After washing several times for 5 min each in PBS-T, the fragments were incubated in the PBS-T containing biotinylated secondary antibody, followed by conventional $\mathrm{ABC}$ methods. The peroxidase reaction was detected by incubating sections in a substrate solution $\left(0.05 \%\right.$ DAB, $0.04 \% \quad \mathrm{H}_{2} \mathrm{O}_{2}$ in Tris $/ \mathrm{HCl}$ buffer, $\mathrm{pH}$ 7.6). After the immunoreactions were completed, specimens were re-fixed with $2 \%$ glutaraldehyde followed by $1 \% \mathrm{O}_{5} \mathrm{O}_{4}$ in $\mathrm{PB}$ and were embedded in Epoxy resin. Ultrathin sections were cut and observed under the TEM(HITACHI
H-7000). Monoclonal anti-PA antibody was from Sigma and reacts specifically with PA. The antiTR-T antibody was purchased from Oncogene Sci., and is reactive with TR-T from species such as rat and rabbit.

In the present study, the immunolabelling was tested by substitution of the anti-PA and anti-TR-T antibody with Texas-Red-labelled streptavidin or FITC-labelled secondary antibody (anti-mouse $\operatorname{IgG}_{1}$ ). No labelling was found in those control experiments.

\section{Results}

The present study has consisted of an immunohistochemical investigation of the localization of PA in cross and longitudinal sections of skeletal muscle fibers, and of the spatial relationship between PA and TR localization in the cytoplasm of muscle fibers. Regarding the antibody against Troponin subfractions, the present authors have used antiTroponin-T purchased from Oncogene Sci., USA, since appropriate anti-Troponin-C antibody was not available. Since Troponin-T and Troponin-C are well known to locate intimately and regularly along with the actin filaments, the estimation of Troponin$\mathrm{T}$ location can be considered to indicate the localization of Troponin-C which directly binds with $\mathrm{Ca}^{2+}$.

\section{Observations of cross sections:}

First of all, the PA-localization and TR-localization in the cross sections of muscle fibers were examined respectively. It was found that the PA-positive immunofluorescence in cross sections of muscle fibers have different intensities (Fig. 1-A). The staining intensity of the skeletal muscle fibers was divided into the following three groups; strong, moderate and unstained. With regard to the muscular fiber types, the strongly immunoreactive fibers were found to be type IIB fibers, the moderate ones were type IIA fibers and the unstained fibers were type I fibers. It was also clear that the immunostaining pattern of the TR-immunoreactive fibers was very similar to the pattern of PA immunoreactivity in the cross sections (Fig. 1-B).

The dual-staining of cross sections with antibodies against both PA and TR demonstrated that all of the PA-immunoreactive fibers were also TR immunoreactive (Fig. 1-C). In other words, the PA-immunoreaction pattern was exactly correlated with fiber typing.

\section{Observations of longitudinal sections:}

The observation of longitudinal sections of skeletal muscle fibers is essential in order to elucidate the distribution and localization of PA within the cyto- 
plasm of the muscle fiber. The fluorescent immunohistochemical studies on the longitudinal sections revealed that the PA was distributed predominantly in the I-bands of the sarcomeres of skeletal muscle fibers and not in the A-bands (Fig. 2-B). In the case of TR, TR-immunoreactivity was demonstrated consistently in the I-band of the sarcomere (Fig. 2-C). Therefore, the I-bands of muscle fibers exhibited not only strong immuno-reactivity to the anti-PA but also to anti-TR. The CLSM image analysis of the specimens which were doubly reacted with both types of antibody clearly showed the PA localized in intimate proximity to TR (Fig. 2-D).

3. Electron microscopic immunohistochemistry:

The ultrastructual findings were in good agreement with those by CLSM. PA-immunoreactive products were seen in I-bands and not A-bands (Fig. 3). They seemed to locate diffusely in the sarcoplasmic matrix throughout the I-band region, showing no particular affinity to the filaments in A-bands.

\section{Discussion}

PA has been considered to be involved in the muscular contraction-relaxation cycle. Previous combined-studies by physiologists and histologists have demonstrated the strong involvement of PA in the relaxation stage of skeletal muscle twitch mechanisms, and the principal interest of those histological researchers seemed to be the relationships between the muscular contractive properties and the fiber types. This may be the main reason why the histological literature concerning PA localization is restricted only to fiber typing. The present study has demonstrated the distribution and localization of the PA in cross and longitudinal sections of skeletal muscle fiber and also clarified the spatial relationship between PA and TR.

Viewing cross sections of muscle fibers immunohistochemically reacted with anti-PA antibody, there were three different fiber types showing a checker-board pattern. According to the previous immunohistochemical studies, the strongest PAimmunoreactive fibers belongs to type IIB fiber, moderate ones to type IIA and the weakest are type I fiber. The dual-staining method produced a TR positive reaction in the fibers which were PA-immunoreactive in the cross section of the muscle fibers. Previous studies, using immunohistochemical methods, described that TR was specifically localized in type II fiber (fast-twitch fiber) and that it was absent in type $I$ fibers ${ }^{20)}$. Thus, it is obvious that a close correlation exists in the localization between the PA and TR-T in the skeletal muscle fibers.
The question which we must consider next is how PA is distributed within the cytoplasm of a singule muscle fiber and, further, how PA is related to TR within the cytoplasm. We have, therefore, undertaken a serious examination of the longitudinal section at both the light microscopic and ultrastructural level. The present fluorescent immunohistochemical studies on the longitudinal sections of skeletal muscle fibers revealed the predominant localization of PA in the I-band of the sarcomeres of each fiber. So, as shown in figure (2-B), the CLSM has clearly depicted PA immunoreactivity especially in the I-band region, not in the A-band, and also revealed a close relationship in the intracytoplasmic localization between PA and TR. The CLSM images clearly showed that PA is located consistently in the I-band of the muscle fiber together with TR. As for the PA distribution in the longitudinal sections of the muscle fibers under the electron microscope, PA-immunoreactivity was revealed mainly in the I-band region of the sarcomeres and was occasionally found to be much stronger in the triad-region and/or Z-band region, relatively decreased in the A-I transitional region and decreased in the A-band. These latter findings were difficult strictly to confirm, however, since the present studies were qualitative, not quantitative.

The contraction-relaxation cycle of the muscle is a $\mathrm{Ca}^{2+}$ exchange between SR and myofibrils ${ }^{1.4 .17)}$. The muscular contraction is initiated by release of $\mathrm{Ca}^{2+}$ from the terminal cisternae of the SR and the relaxation is achieved by the retrieval of $\mathrm{Ca}^{2+}$ from TR-C to the PA after which the ions are retained in the SR. It seems reasonable, therefore, that the PA should be located predominantly in the I-band of the muscle fiber. The mode of distribution of PA may facilitate the calcium-movement in the muscle fiber during the contraction-relaxation cycle. This supposition may be compatible with the findings that the fast twitch muscle fiber contains high concentrations of PA.

\section{References}

1) Heizmann $\mathrm{CW}$. Parvalbumin, an intraccllular calciumbinding protein: distribution, properties and possible roles in mammalian cells. Experientia 1984; 40:910-921.

2) Heizmann $\mathrm{CW}$ and Celio MR. Immunolocalization of Parvalbumin. Methods Enzymol 1987: 139:552-570.

3) Heizmann CW, Berchtold MW and Rowlerson AM. Correlation of parvalbumin concentration with relaxation speed in mammalian muscle. Proc Natl Acad Sci USA 1982: 79:7243-7247.

4) Fuchtbauer EM, Rowlerson AM, Gotz K. Fricdrich G. Mabuchi K, Gergely J and Jockusch H. Direct correlation of Parvalbumin levels with myosin isoforms and succinate dehydrogenasc activity on frozen sections of rodent muscle. J Histochem Cytochem 1991: 39:355-361. 
5) Leclerc E, Leclerc L, Cassoly R, Terrossian ED, Wajcman H, Poyart $C$ and Marden MC. Heme binding to calmodulin, troponin $\mathrm{C}$, parvalbumin, as a probe of calcium-dependent conformational changes. Arch Biochem Biophys 1993; 306: $163-168$.

6) Klug G, Reichmann $\mathrm{H}$ and Pette D. Rapid reduction in parvalbumin concentration during chronic stimulation of rabbit fast twitch muscle. FEBS Lett 1983; 152:180-182.

7) Ohtuki I. Localization of troponin in thin filament and tropomiosin paracrystal. J Biochem 1974; 75:753-765.

8) Anderson JE, Bressler BH and Ovalle WK. Functional regencration in the hindlimb skeletal muscle of the $\mathrm{mdx}$ mouse. J Muscle Res Cell Motil 1998; 9:499-515.

9) Shima $K$, Tashiro $K$, Tsukada $Y$ and Hirai H. Carbonic anhydrasc-1II immunohistochemical localization in human skeletal muscle. Acta Neuropathol 1983; 59:237-239.

10) Marion S, Prince E and Leberer E. Parvalbumin in mouse muscle in vivo and in vitro. Differenation 1989; 40:10-16.

11) Muntener M, Rowlerson AM, Berchtold MA and Heizmann $\mathrm{CW}$. Changes in the concentration of the calcium-binding parvalbumin in cross-reinnervated rat muscles. $J$ Biochem 1987: 262:465-469.

12) Muntener $M$. Hardeveld $C$, Everts $M$ and Heizmann $C W$. Analysis of $\mathrm{Ca} 2+-$ binding parvalbumin in rat skcletal muscles of different thyroid states. Exp Neurol 1987; 98: $529-541$.

13) Imaizumi $M$ and Tanokura $M$. Heat capacity and entropy changes of troponin $C$ from bullfrog skeletal muscle induced by calcium binding. Eur J Biochem 1990; 192:275-281.

14) Celio MR and Heizmann $\mathrm{CW}$. Calcium-binding protein parvalbumin as a ncuronal marker. Nature 1981; 293: 300-302.

15) Cclio MR and Heizmann CW. Calcium-binding protein parvalbumin is associated with fast contracting muscle fibers. Naturc 1982: 297:504-506.

16) Pauls TL, Cox JA, Heizmann CW and Hermann A. Sarcoplasmic calcium-binding proteins in aplysia nerve and muscle cells. Eur J Neurosci 1993; 5:549-559.

17) Schmitt TL and Pettc D. Fiber type-specific distribution of parvalbumin in rabbit skeletal muscle. Histochemistry 1993; 96:459-465.

18) Endo T, Kobayashi M, Kobayashi $S$ and Onaya T. Immunocytochemical and biochemical localization of parvalbumin in the retina. Cell Tissuc Res 1986; 243:213-217.

19) Ogawa $Y$ and Tanokura $M$. Steady-state properties of calcium binding to parvalbumins from bullfrog skeletal muscle: Effect of $\mathrm{Mg}^{2+}, \mathrm{pH}$, ionic strength, and temperature. $\mathrm{J}$ Biochem 1986; 99:73-80.

20) Onoyama Y. Effect of antibodics against troponin components on the Actomyosin ATPase activity of fetal and adult skeletal muscle of rabbit. Fukuoka Acta Med 1986; 77:419-426.

21) Ito $Y$, Sato $T$ and Tauchi $H$. Quantitative immunohistochemistry of parvalbumin expression in the rat diaphragm. Analyt Quant Cytol Histol 1993; 15:201-205.

22) Inaguma $Y$, Kurobe $N$, Shinohara $H$ and Kato $K$. Sensitive immunoassay for rat parvalbumin: tissue distribution and developmental changes. Biochemica et Biophysica Acta 1991: 1075:68-74.

\section{Explanation of Figures}

\section{Plate I}

Fig. 1-A. A cross section of skeletal muscle (the extensor digitorum longus) showing the positive immunoreactivity against PA.

Fig. 1-B. Immunostaining of the same section as Fig 1-A with anti-TR.

Fig. 1-C. Dual-staining pattern of the section which was reacted with antibody against PA and TR. The PA-immunopositive reaction was colored red and the TR-immunopositive reaction was colored green by means of the CLSM imaging analyzer.

Fig. 2-A. Phase contrast microscopy: the longitudinal section of a sigule muscle fiber of the extensor digitorm longus.

Fig. 2-B. A longitudinal section of the muscle fiber reacted with anti-PA antibody.

Fig. 2-C. TR immunoreactivity in the longitudinal section of the skeletal muscle fiber.

Fig. 2-D. A combined-phase contrast and fluorescent image of the longitudinal section. The PA-immunopositive reaction is colored red and the TR-immunopositive reaction green using CLSM image analyzer. Both PA and TR were located consistently in the I-band of the muscle fiber. 


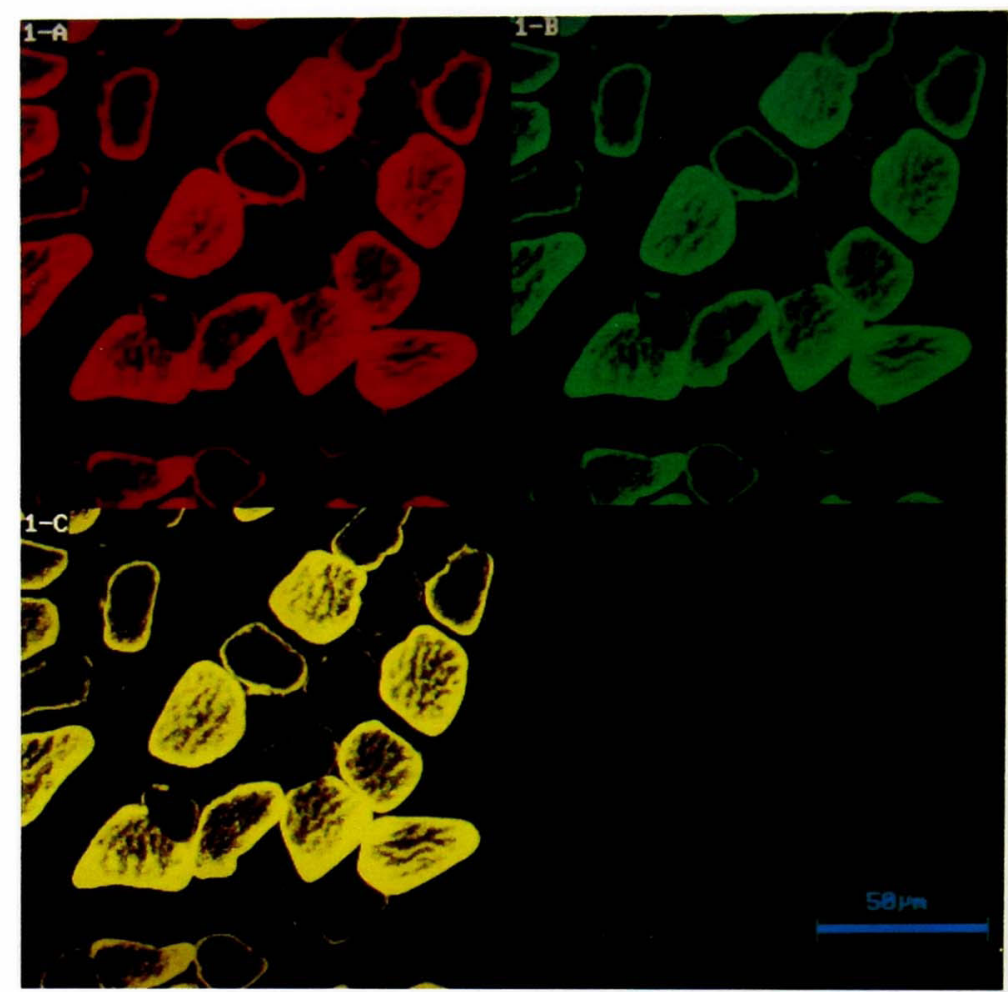

Fig1

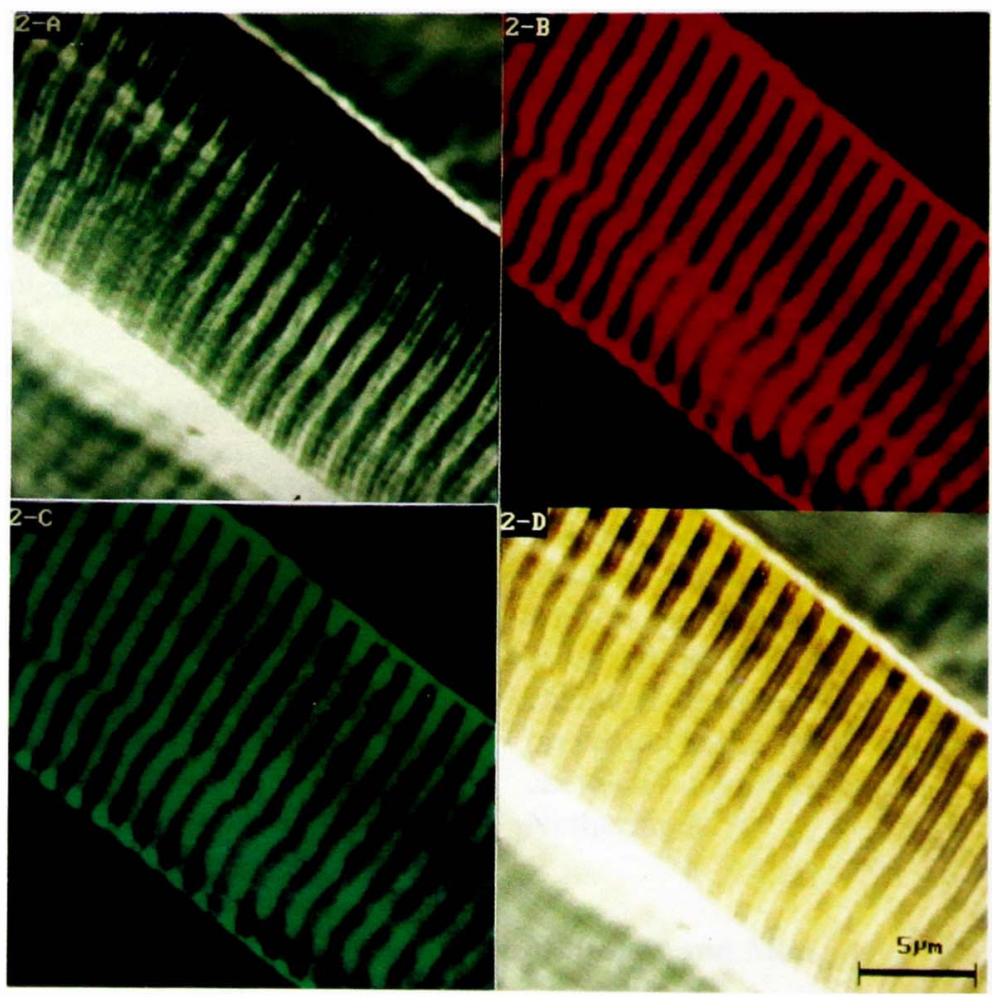

Fig 2 
Plate II

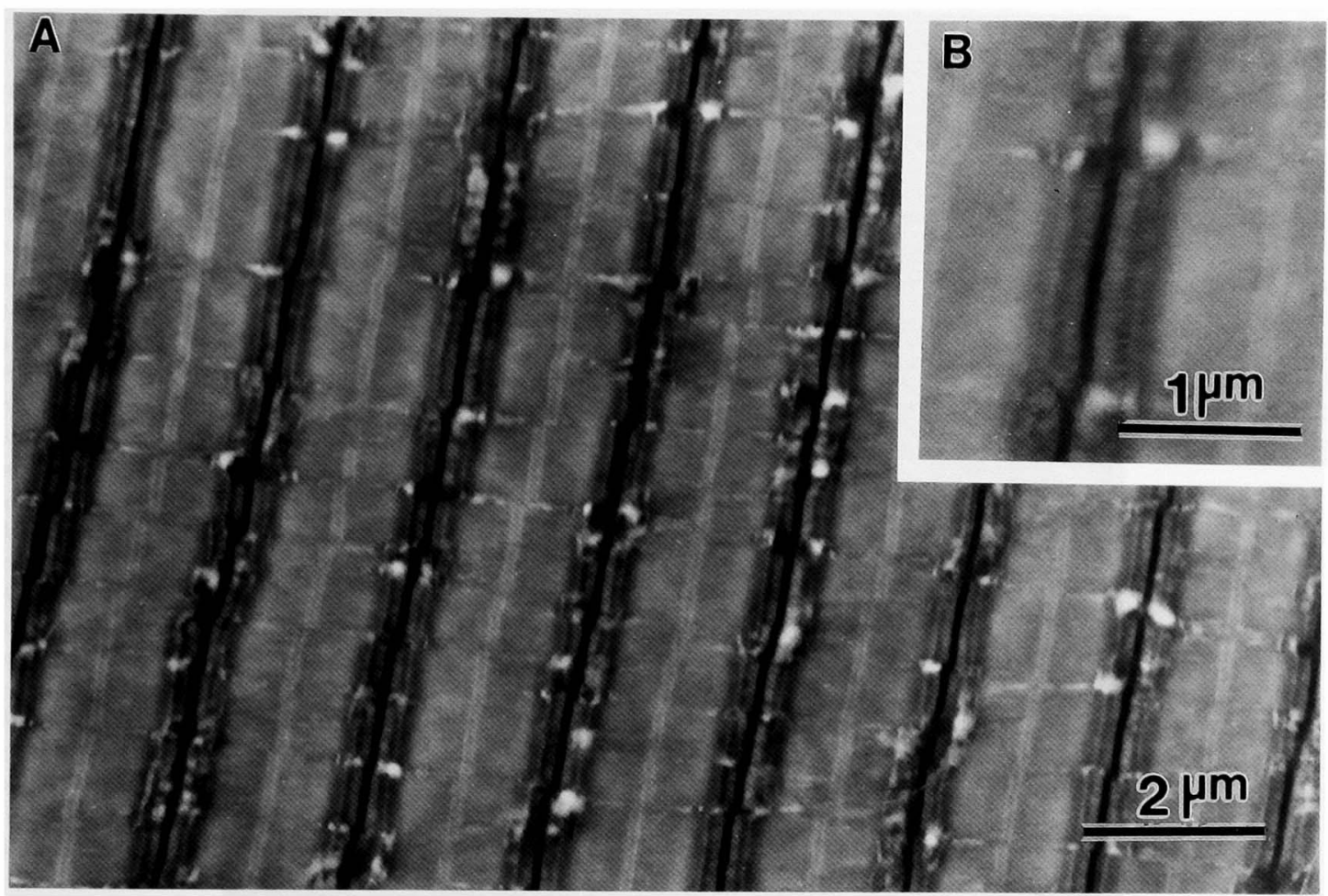

$\mathrm{Fig} \mathbf{3}$

\section{Plate II}

Fig. 3-A. PA-immunorcactivity in a skelctal muscle fiber which was scen under TEM.

Fig. 3-B. A closer view of the immunoreactive portion of the longitudinal section. 